\title{
APPROXIMATE SOLUTIONS TO SEVERAL VISIBILITY OPTIMIZATION PROBLEMS*
}

\author{
ROSTISLAV GOROSHIN ${ }^{\dagger}$, QUYEN HUYNH ${ }^{\ddagger}$, AND HAO-MIN ZHOU ${ }^{8}$
}

\begin{abstract}
The visibility level set function introduced by Tsai et al. allows for gradient based and variational formulations of many classical visibility optimization problems. In this work we propose solutions to two such problems. The first asks where to position $n$-observers such that the area visible to these observers is maximized. The second problem is to determine the shortest route an observer should take through a map such that every point in the map is visible from at least one vantage point on the route. These problems are similar to the "art gallery" and "watchman route" problems, respectively. We propose a greedy iterative algorithm, formulated in the level set framework as the solution to the art gallery problem. We also propose a variational solution to the watchman route problem which achieves complete visibility coverage of the domain while attaining a local minimum of path length.
\end{abstract}

Key words. Visibility, optimization, level set method.

AMS subject classifications. $65,49,35$.

\section{Introduction}

Visibility is the mathematical abstraction that divides a domain $(\Omega)$ populated with occluders into visible and invisible regions as observed from a vantage point $\left(x_{o}\right)$ in the domain. For our purposes, an observer is omnidirectional and views the scene along rays emanating from $x_{o}$, which represent lines of sight. In this work we restrict ourselves to a bounded, two-dimensional, planar domain. We assume that the map of the domain is known a priori (unexplored domains are discussed in [7]). Visibility optimization usually refers to maximizing the visible region in a constrained optimization problem, therefore visibility optimization is a set cover problem [6]. In this work we will address two such problems:

1. What is the minimum number of observers required to cover the entire domain, and where must they be placed?

2. What is the shortest path through the domain such that every point in the domain is visible from at least one point on the path?

These problems resemble the classical "art gallery" and "watchman route" problems, respectively $[10,8,5]$. In both of these problems our primary goal will be to achieve complete visibility coverage of the domain. Due to the non-convex nature of these problems gradient ascent methods are inadequate in achieving globally maximal visibility coverage [4]. Therefore our goal is to introduce computationally tractable solutions which achieve complete visibility coverage of the domain while achieving only locally optimal performance in other metrics. For example, our solution to the second problem is guaranteed to attain maximal visibility coverage of the domain, by

*Received: March 9, 2010; accepted (in revised version): September 24, 2010. Communicated by Shi Jin.

${ }^{\dagger}$ Courant Institute of Mathematical Sciences, New York University, New York 10003 (goroshin@ cs.nyu.edu). On leave from Naval Surface Warfare Center Panama City Division.

${ }^{\ddagger}$ Naval Surface Warfare Center Panama City Division, 110 Vernon Avenue, Panama City, Florida, 32407-7001 (quyen.huynh@navy.mil).

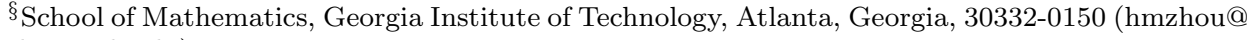
math.gatech.edu). 
construction, while achieving only a local minimum of path length. For many applications the importance of attaining complete sensor coverage far outweighs secondary objectives of minimizing the number of sensors in the first problem and minimizing the route length in the second problem [9].

The curve separating the visible regions from the invisible regions is called the visibility boundary, or shadow boundary. Classically, visibility boundaries have been computed using computationally intensive ray tracing techniques [1]. Tsai, Osher, et al. recast the visibility computation into a boundary value problem and used a numerical PDE solver called "fast sweeping" to compute a numeric approximation of visibility boundaries. The fast sweeping computation produces an embedding of codimension-one of the approximate visibility boundary, called the visibility level set function [14]. As we will see, this implicit representation of the visibility boundary has many well known advantages popularized by level set methods [11]. The analytic expression for the visibility level set function can be written as

$$
\Phi\left(x ; x_{0}\right)=\min _{z \in L\left(x, x_{0}\right)} \Psi(z),
$$

where $\Psi$ is a signed distance embedding of the occluders defined over the domain such that $\Psi>0$ outside the occluders, and $L\left(x, x_{0}\right)$ is the line segment connecting the observer stationed at $x_{o}$ to a point $x$. If the value of $\Phi$ is negative at $x$ then the point $x$ is occluded from the observer at $x_{0}$. Points where $\Phi=0$ are at the edge of visibility, these points constitute the visibility boundary with respect to $x_{o}$. The visibility level set function is a real valued function which measures the largest depth of occlusion (if $x$ is occluded), or the shortest distance from the ray to the nearest occluder (if $x$ is visible); see Figure 1.1. This property is crucial for enabling gradient-based and variational visibility optimization as introduced by Cheng et al. in [4].

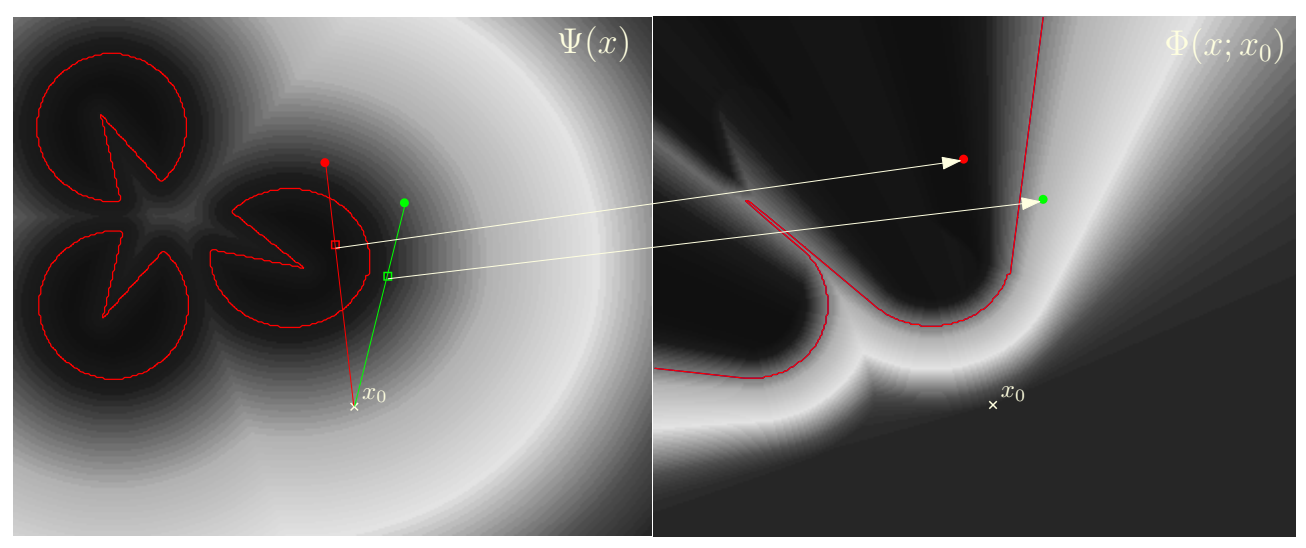

Fig. 1.1: The visibility level set function (right) and signed distance embedding of the occluders (left) for fixed $x_{0}$. 


\section{Finite-dimensional visibility optimization problems}

In the level set framework the observed visible volume for a single observer is defined as

$$
V\left(x_{0}\right)=\int_{\Omega} H\left(\Phi\left(y ; x_{o}\right)\right) d y
$$

where $H(\cdot)$ is the one-dimensional Heaviside step function, with $H(0)=0$. Visibility optimization problems generally seek to maximize this volume.

We can extend this definition to multiple observers and define the joint visible volume of $n$ observers as

$$
V\left(x_{1}, x_{2}, \ldots, x_{n}\right)=\int_{\Omega} H\left(\sum_{i=1}^{n} H\left(\Phi\left(y, x_{i}\right)\right)\right) d y .
$$

Finally, we may define the visible volume seen by a continuum of observers located on a parameterized closed ${ }^{1}$ curve $C(s)$ as

$$
V(C(s))=\int_{\Omega} H\left(\int_{C} H(\Phi(y ; C(s)) d s) d y .\right.
$$

The above expression may also be interpreted as the cumulative visible volume covered by an observer traversing the curve $C(s)$. Similar notions were defined by Cheng et al. [4].

The visible volume of observers is optimized over their vantage points $x \in \Omega$. Discrete optimization problems defined by (2.1) and (2.2) will be discussed in this section. Equation (2.3) is a functional and is optimized using variational techniques over the curve $C(s)$, usually while imposing other constraints on the curve. The variational problem relating to (2.3) will be discussed in the next section.

In [4] Cheng et al. propose a gradient ascent algorithm for maximizing the visible volume of an observer by allowing the observer to move in the direction specified by a numerical implementation of the partial differential equation

$$
\partial_{t} x_{0}=\nabla_{x_{0}} V\left(x_{0}\right)
$$

The above equation may be regarded as the gradient ascent on the surface $V\left(x_{0}\right)$. This surface may be constructed by evaluating Equation (2.1) at all possible observer locations. That is, for each point in the domain $(\Omega)$ we assign the value of the integral in (2.1). The visible volume function for a single observer is a mapping which takes the form

$$
V: \Omega \subset \mathbb{R}^{2} \mapsto V\left(x_{0}\right) \in \mathbb{R}^{+} .
$$

The surface is shown in Figure 2.1 with its level curves. The brightness at each point is proportional to the area visible to an observer stationed at that location. The single observer placement problem reduces to finding the global maximum on this surface. The trajectory of an observer moving according to (2.4) is normal to the level lines of the visible volume surface; see [4]. However, due to the non-convexity

\footnotetext{
${ }^{1}$ The curve may be open; however for ease of implementation in the level set framework we restrict ourselves to closed curves in this work.
} 


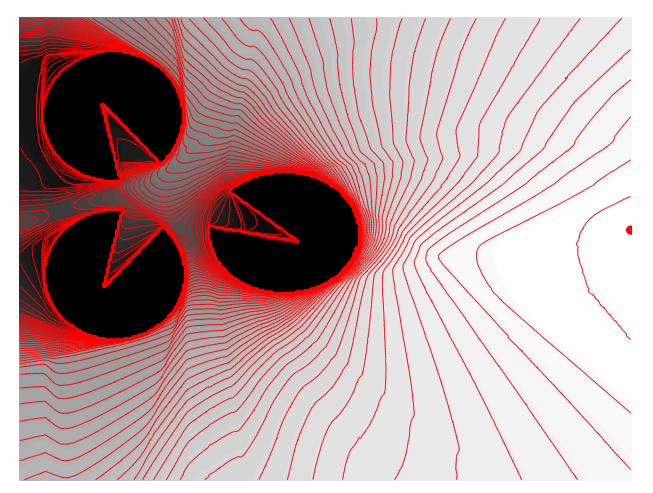

Fig. 2.1: The visible volume function with its level-curves.

of the visible volume function there is no guarantee that the observer will arrive at a globally optimal observer location (red point on far right).

Global optimization methods should be used to find an optimal observer position. The computational complexity of the fast sweeping algorithm for each $x_{o}$ scales linearly with the number of grid points $(N)$ [14], so that it is computationally feasible to perform exhaustive search in order to determine the globally optimal solution for the single observer placement problem. The overall exhaustive search computation over the observer grid scales as $O\left(N^{3}\right)$. Coarse to fine searches can be used to accelerate the process in large domains.

Multiple observer visibility optimization seeks to maximize the visible volume seen jointly by $n$ observers. In general, the visible volume in a bounded domain is bounded between

$$
0 \leq V \leq \int_{\Omega} H(\Psi(y)) d y
$$

The art gallery problem seeks to cover the entire domain, excluding the area occupied by the occluders, with a minimum number of observers. To cover the entire domain with $n$ observers is to achieve equality in the upper bound of (2.5) by maximizing (2.2) over the observer locations. The solution to the art gallery problem must satisfy

$$
\int_{\Omega} H\left(\sum_{i=1}^{n} H\left(\Phi\left(y, x_{i}\right)\right)\right) d y=\int_{\Omega} H(\Psi(y)) d y .
$$

In fact, for the art gallery problem the above condition is equivalent to

$$
H\left(\sum_{i=1}^{n} H\left(\Phi\left(y, x_{i}\right)\right)\right)=H(\Psi(y)) .
$$

It is useful to define the fraction of the domain visible to (covered by) the $n$ observers as

$$
V_{\text {norm }}:=\frac{\int_{\Omega}\left(\sum_{i=1}^{n} H\left(\Phi\left(y, x_{i}\right)\right)\right) d y}{\int_{\Omega} H(\Psi(y)) d y}
$$


We call $V_{\text {norm }}$ the normalized visible volume; it is a useful quantity for measuring the convergence of visibility optimization algorithms. Analogous measures can be defined for Equations (2.1) and (2.3). Note that $0 \leq V_{\text {norm }} \leq 1$.

The problem of multiple observer optimization is much larger than single observer optimization [10]. The function defined by (2.2) is a mapping of the form

$$
V: \Omega^{n} \subset \mathbb{R}^{2 n} \mapsto V\left(x_{1}, x_{2}, \ldots, x_{n}\right) \in \mathbb{R}^{+} .
$$

As before, we discretize the domain into $N$ regularly spaced grid points. The computational complexity of exhaustive search for $n$-observer placement is of order $O\left(N^{n}\right)$. Even if the visibility level set function is computed a priori at each grid point, exhaustive search through all possible placements for more than a few observers becomes computationally prohibitive. On the other hand, gradient ascent approaches, such as those found in [4], are prone to local maxima and will require at least as many observers as exhaustive search to cover an area. A good compromise is to avoid gradient based optimization altogether and perform iterative exhaustive searches in a reduced space.

We propose an alternating maximization scheme for multiple observer optimization. For each observer, indexed by $j$, the globally optimal solution to

$$
\underset{x_{j} \in \Omega}{\arg \max } \int_{\Omega} H\left(H\left(\Phi\left(y ; x_{j}\right)\right)-H\left(\sum_{\substack{i=1 \\ i \neq j}}^{n} H\left(\Phi\left(y ; x_{i}\right)\right)\right) d y\right.
$$

is obtained by computing (2.7) at every grid point outside of the occluders. The first term in the above expression represents the visible region seen by the $j^{\text {th }}$ observer stationed at $x_{j}$. The second term is the joint visible region of all other observers. The scheme exhaustively searches for the optimal location of one observer at a time, discounting the region visible to already placed observers. The process is repeated for each observer. The optimal observer positions may not be unique, therefore an optimal position is chosen at random in order to avoid repeating the iteration. Once all observers have been positioned in this way, we proceed to the second iteration by removing an observer and repositioning it by solving (2.7) again. Again, observers are updated in random order in order to avoid repeating the iteration. This process is repeated for all observers until the normalized visible volume seen by the observers (2.6) converges to some value. This algorithm is similar to the heuristic algorithm for finding a set cover from finite subsets proposed in [6]. The pseudocode for multiple observer optimization is given in Algorithm 1.

The algorithm is illustrated in Figure 2.2. In this example we initialize the observer positions by solving (2.7), although the observer positions can be initialized arbitrarily without greatly increasing the number of iterations required for convergence. The first observer is positioned by solving (2.7) with only one observer, making the second term in (2.7) zero. This is how the visible region A (in light gray) is obtained in Figure 2.2(a). Next, the second observer is positioned to maximize the visible volume not contained within A, again by solving (2.7); this time the second term will correspond to the visible region seen by the first observer $(\mathrm{A})$. The third observer is positioned to maximize the visible region not contained within $A \cup B$. This process is repeated in the second iteration. One way to ensure that the entire domain is covered 


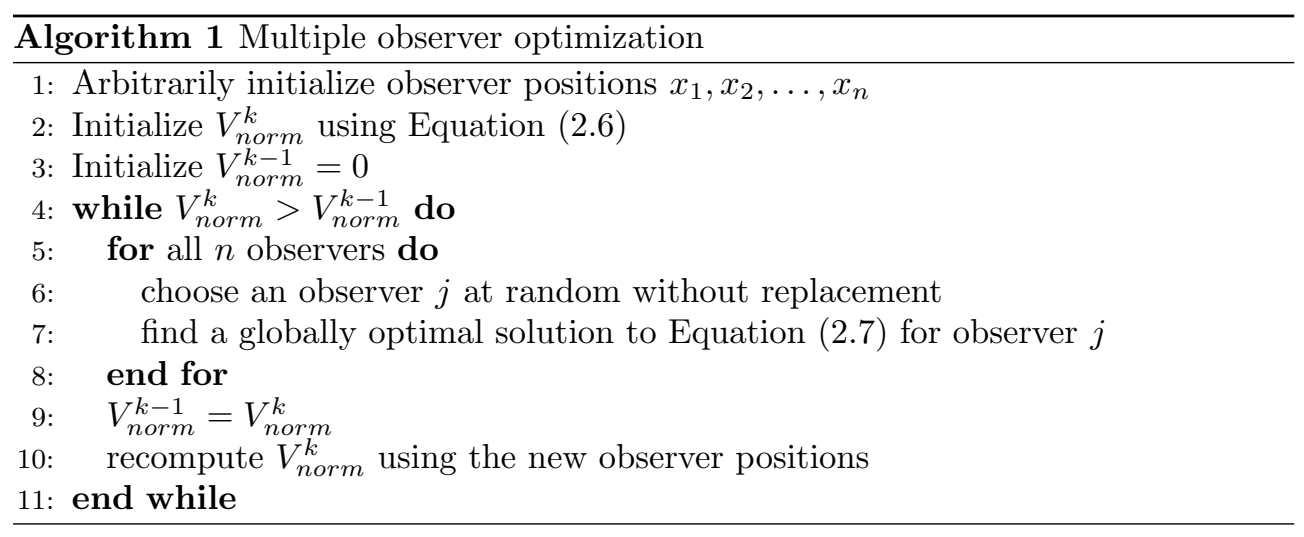

(i.e. to solve the art gallery problem) is to increment the number of observers and repeat the placement algorithm if $V_{\text {norm }}<1$ after convergence.

By precomputing the visibility level set function at all grid locations it is possible to reduce the computational complexity of evaluating (2.7) at every grid location in order to find the optimal observer position. This eliminates the need to compute $\Phi$ within the loop of the above algorithm. The resulting process requires at most $n\left(N^{2}+N(n-1)\right)$ addition/subtraction operations per iteration.

Figure 2.3(b) shows the result of the final iteration of the placement of six observers using the proposed procedure. The entire map could not be covered with only six observers. Figure 2.3(d) depicts the resulting placement found in the final iteration of the algorithm for eight observers on the same map. Figure 2.3(c) depicts the surface defined by (2.7) for the positioning of the $7^{\text {th }}$ observer. Figure 2.3(a) depicts the percent visibility as a function of the observer repositioned. The first observer placed covers approximately $55 \%$ of the map, the first two cover approximately $77 \%$, at the end of the first iteration (first red point) the observers cover over $99 \%$ of the map. It is clear that the visible volume must be increasing as the observers are repositioned to more optimal locations. We have also noted that the visible volume function is bounded, as given by (2.5). The visible volume function can therefore be regarded as a bounded increasing sequence, which must converge. Therefore the multiple observer placement algorithm must converge. However, for a fixed number of observers the algorithm does not necessarily converge to a globally optimal nor a unique solution.

\section{Variational visibility optimization}

Until now we have only considered finite dimensional visibility problems. Many practical visibility based problems require variational formulations; the watchman route problem discussed in the introduction is only one example. We will approach the watchman route problem by representing the various objectives involved using energy functionals. The inspection route itself will be represented by an implicit curve using the level set representation $[12,11]$. We will consider only closed curves mainly due to their simple representation in the level set framework. We note that many practical applications, for example computing optimal patrol routes, require closed paths. Implementation issues will be discussed in in the next section.

The authors in [4] evolved parameterized curves according to gradient ascent on the Euler-Lagrange equation of a visibility based functional. In this section we define a new visibility functional and introduce a variational solution to the watchman route 


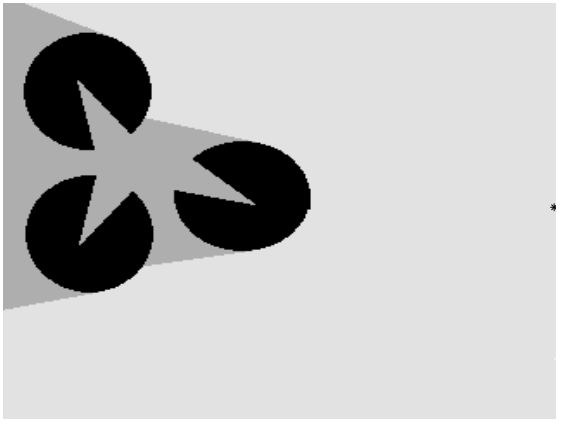

(a) $A$

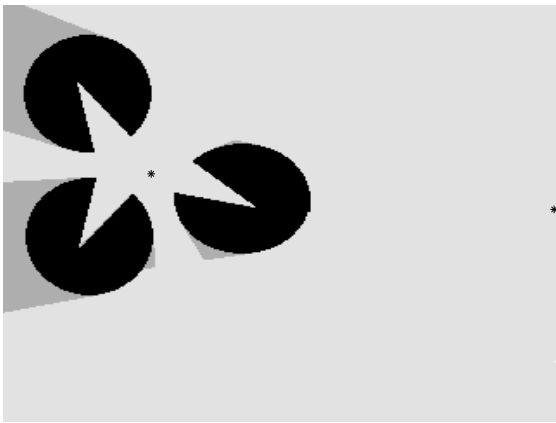

(c) $A \cup B$

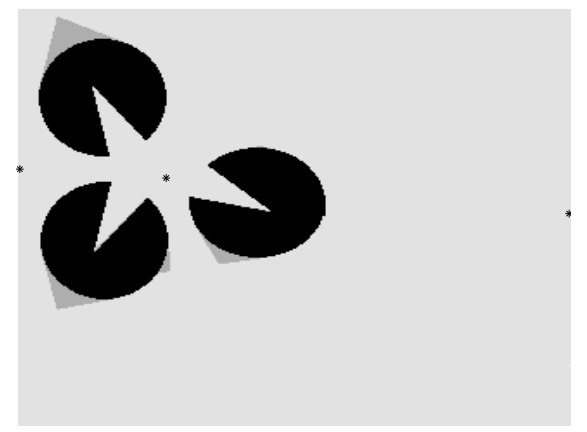

(e) $A \cup B \cup C$ first iteration

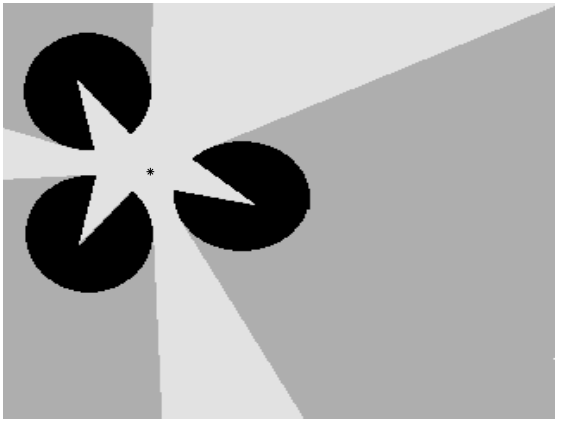

(b) $B$

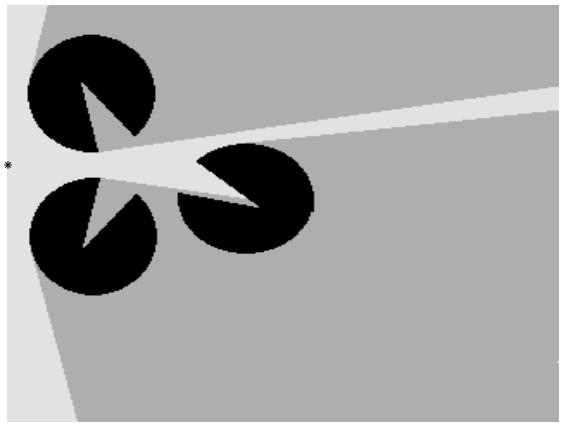

(d) $C$

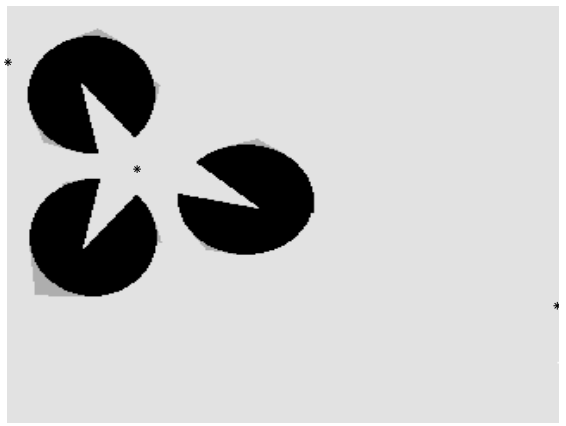

(f) $A \cup B \cup C$ fourth and final iteration

Fig. 2.2: Multiple Observer Placement Algorithm.

problem. Unlike the work in [4] which disregards global optimality, our goal will be to formulate a solution to the watchman route problem which achieves complete visibility coverage of the domain. We will discuss how to initialize and evolve the route in order to avoid local visibility extrema and achieve complete visibility coverage. Before proceeding we note that in the computational geometry literature the watchman route problem is defined for domains with polygonal boundaries [10, 13]; here we do not make any assumptions about the geometry or topology of the domain. Nevertheless we refer to this problem as the watchman route problem. 


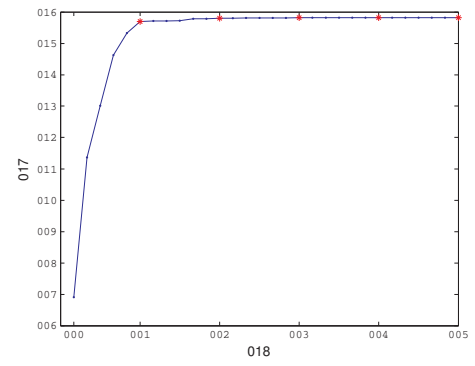

(a)

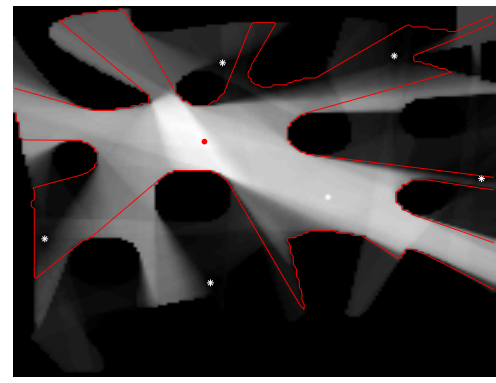

(c)

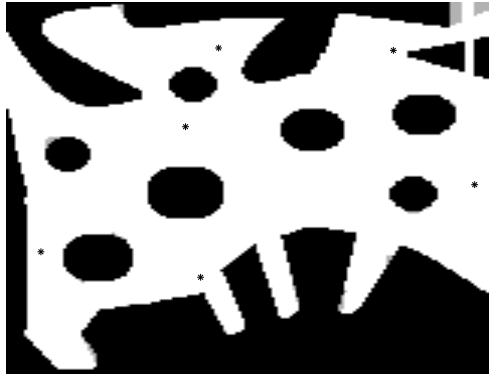

(b)

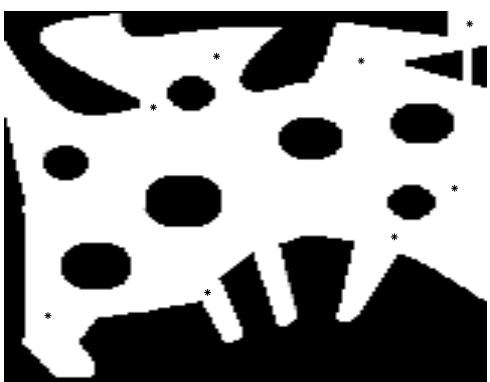

(d)

Fig. 2.3: Multiple observer placement in a complex environment.

In the previous section we defined the cumulative visible volume covered by an observer traversing the curve $C(s)$ by Equation (2.3). We may rewrite (2.3) in a more compact form using the concept of exposure introduced in [4]. Exposure is a useful quantity in visibility problems for observers with memory. Consistent with the notation of [4], the exposure is defined as

$$
\mathcal{X}(x ; C)=\int_{C} H(\Phi(x ; C(s))) d s,
$$

where $s$ is the arc length parameter. The exposure may be interpreted as the length of time spent observing the point $x$ while traversing the curve $C$ at unit speed[4]. Using (3.1) we rewrite the functional defined by (2.3) in a more compact form:

$$
V(C)=\int_{\Omega} H(\mathcal{X}(y ; C)) d y .
$$

Our motivation for defining (3.2) is to measure the visible area seen from the curve $C(s)$. In contrast, [4] defined a visibility based functional which measures the deviation of the exposure from some constant value $(A)$, for all points in the domain outside of the obstacles $(D)$. We restate their functional for comparison:

$$
\int_{\Omega \backslash D}(\mathcal{X}(y ; C)-A)^{2} d y .
$$

Because the exposure is a positive semidefinite function, (3.2) exactly corresponds to 
the zero-norm of the exposure. On the other hand, the above functional is the square of the two-norm of the exposure.

We call the variational derivative of Equation (3.2) with respect to the curve $C(s)$ the velocity field due to visibility coverage $\left(\vec{v}_{v i s}\right)$, or the visibility velocity field for short. The visibility coverage attained by the curve may be locally maximized by performing gradient ascent on the curve using the visibility velocity field. The following lemma will be used to compute $\vec{v}_{v i s}$.

Lemma 3.1. It has been shown in [3] that a functional which takes the form

$$
E(C)=\int_{C} W d s
$$

has the first variation

$$
\frac{d E}{d t}=\int_{C}((\nabla W \cdot \vec{N}) \vec{N}-W \kappa \vec{N}) C_{t} d s
$$

where $\kappa$ and $\vec{N}$ are the curvature and inward unit normal, respectively, of the curve C. Gradient ascent may be performed on the curve using the corresponding EulerLagrange equation

$$
\frac{d C}{d t}=(\nabla W \cdot \vec{N}) \vec{N}-W \kappa \vec{N}
$$

Applying (3.3) to the functional (3.2) we obtain

$$
\begin{aligned}
\frac{d C}{d t}= & {\left[\int_{\Omega} \delta\{\mathcal{X}(y ; C)\} \delta\{\Phi(y ; C(s))\} \nabla_{x_{0}} \Phi(y ; C(s)) \cdot \vec{N} d y\right] \vec{N} } \\
& -\left[\int_{\Omega} H\{\mathcal{X}(y ; C) d y\}\right] \kappa \vec{N} .
\end{aligned}
$$

Because the Heaviside function is positive semi-definite and $\vec{N}$ is the inward normal, the curvature term in the above expression will be in the direction of convexity when it is not null, making it numerically unstable [11]. Therefore, we disregard the curvature term in Equation (3.4). Thus the resulting expression will be denoted as $\vec{v}_{v i s}$ :

$$
\vec{v}_{v i s}=\left[\int_{\Omega} \delta\{\mathcal{X}(y ; C)\} \delta\{\Phi(y ; C(s))\} \nabla_{x_{0}} \Phi(y ; C(s)) \cdot \vec{N} d y\right] \vec{N} .
$$

The resulting equation expresses how the curve $C$ should be deformed in order to optimally increase visibility coverage (via advection), measured by the functional in (3.2). The term $\delta\{\mathcal{X}(y ; C)\}$ is a line integral along the entire curve which represents areas of the domain that are not visible from anywhere on the curve. The term $\delta\{\Phi(y ; C(s))\}$ determines if the point $y$ is at the edge of visibility from the vantage point $C(s)$ on the curve. Finally, the term $\nabla_{x_{0}} \Phi(y ; C(s))$ denotes the gradient of the visibility level set function with respect to the observer position. This is the direction in which the observer at $C(s)$ should move in order to increase visibility of the point $y$. We will use the following lemma about $\vec{v}_{v i s}$ later in our discussion. 
Lemma 3.2. $\vec{v}_{v i s}=0$ inside the occluders.

To see this, consider a point $C\left(s_{0}\right)$ on the curve which is inside an occluder, i.e. $\Psi\left(C\left(s_{0}\right)\right)<0$ or $H\left(-\Psi\left(C\left(s_{0}\right)\right)\right)=1$. Now consider the term $\delta\{\Phi(y ; C(s))\}$ in the definition of $\vec{v}_{v i s}$ at the point $C\left(s_{0}\right)$ and recall the definition of $\Phi$ from Equation (1.1).

$$
\Phi\left(y ; C\left(s_{0}\right)\right)=\min _{z \in L\left(y, C\left(s_{0}\right)\right)} \Psi(z) \leq \Psi\left(C\left(s_{0}\right)\right)<0 .
$$

Therefore $\delta\left\{\Phi\left(y ; C\left(s_{0}\right)\right)\right\}=0$ for all $y$, and thus $\vec{v}_{v i s}=0$.

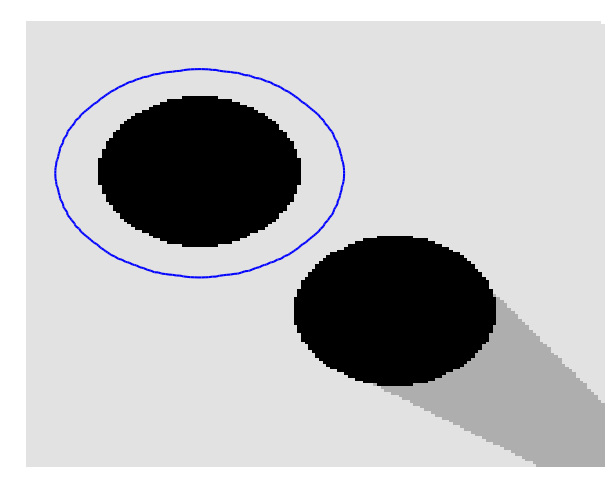

(a) Initial

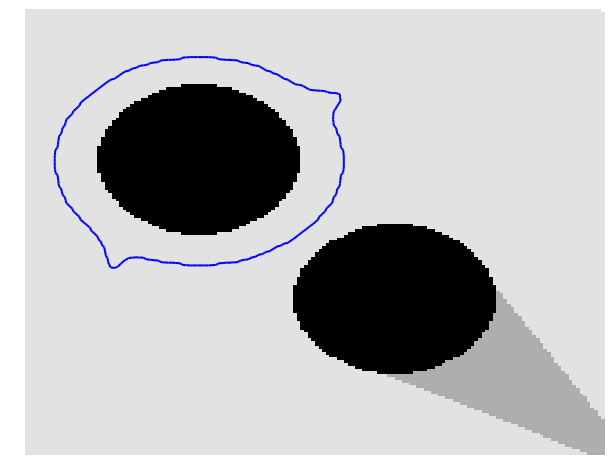

(b) 30 Iterations

Fig. 3.1: Action of Visibility Velocity Field on a Curve.

Figure 3.1 shows the result of allowing a curve (blue circle) to evolve under $\vec{v}_{v i s}$ for several iterations. Note that the curve deforms only at those locations which will optimally increase visibility coverage. As in many gradient based optimization applications initialization is crucial. Complete visibility coverage cannot be attained by evolving an arbitrarily initialized curve under $\vec{v}_{v i s}$ in all but the most basic, convex cases.

Recall that in addition to achieving total visibility coverage we require that the route not pass through any occluders, which also act as obstacles, and be as short as possible. Since (3.2) does not take path length or intersections with occluders into account, $\vec{v}_{v i s}$ will not minimize these other objectives. These constraints are treated as separate penalty energy functionals and have their own corresponding gradient descent velocity field. After discussing obstacle avoidance and route shortening we will introduce a way to combine these constraints with the visibility coverage objective.

If the obstacles are embedded in a signed distance function $\Psi$ such that $\Psi>0$ outside the occluders, then the following energy functional imposes a penalty on those portions of the curve which intersect with the occluders:

$$
E_{o}(C)=\int_{C} H(-\Psi)(\Psi) d s .
$$

The corresponding gradient ascent on the curve is obtained by applying (3.3) to (3.6):

$$
\begin{aligned}
\frac{d C}{d t} & =([-\delta(-\Psi) \Psi \nabla \Psi+H(-\Psi) \nabla \Psi] \cdot \vec{N}) \vec{N}-H(-\Psi) \Psi \kappa \vec{N} \\
& =(H(-\Psi) \nabla \Psi \cdot \vec{N}) \vec{N}-H(-\Psi) \Psi \kappa \vec{N} .
\end{aligned}
$$


The above expression was simplified using the fact that $\delta(-\Psi) \Psi=0$.

The last objective in the watchman route problem is to make the path as short as possible outside the obstacles. This is given by the arc length functional whose corresponding gradient descent is the well known curvature flow:

$$
\begin{aligned}
E_{L}(C) & =\int_{C} d s, \\
\frac{d C}{d t} & =\kappa \vec{N} .
\end{aligned}
$$

We combine (3.7) and (3.8) into a single velocity field, called the path planning velocity field:

$$
\vec{v}_{p}=H(-\Psi)([\nabla \Psi \cdot \vec{N}] \vec{N}-\Psi \kappa \vec{N})+H(\Psi) \kappa \vec{N} .
$$

Note that (3.8) is applied only to portions of the curve outside the obstacles.

The action of the path planning gradient descent velocity field acting on a curve is shown in Figure 3.2. The curve initially intersects the obstacles. Due to the first term in Equation (3.9) the curve moves out of each obstacle in the direction specified by the gradient of the signed distance function. Only curvature flow has any effect on the curve outside the obstacles; it contracts the curve as much as possible without increasing intersections with the obstacles.

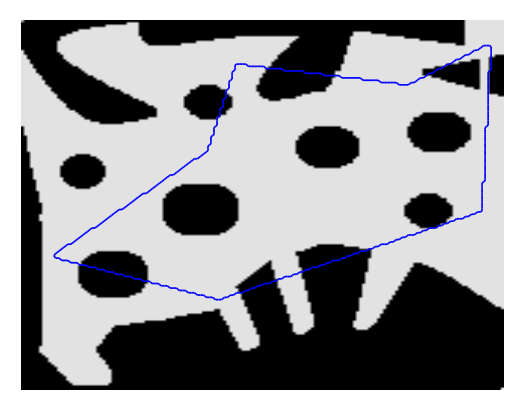

(a) Initial

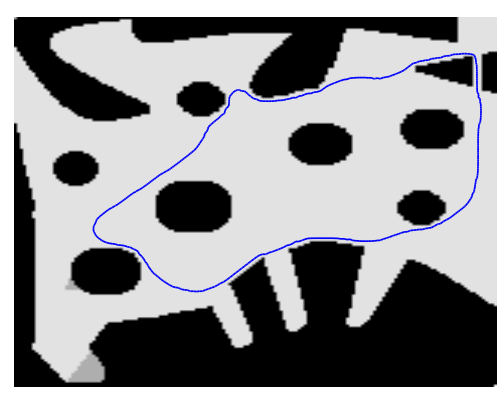

(c) 400 Iterations

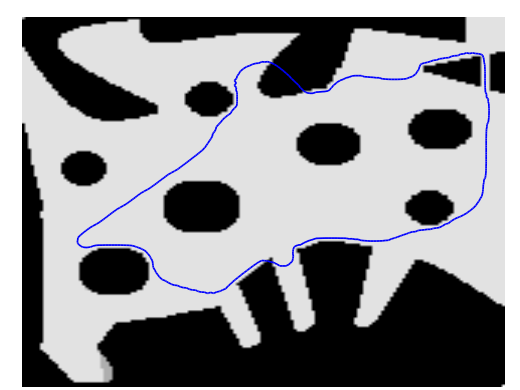

(b) 100 Iterations

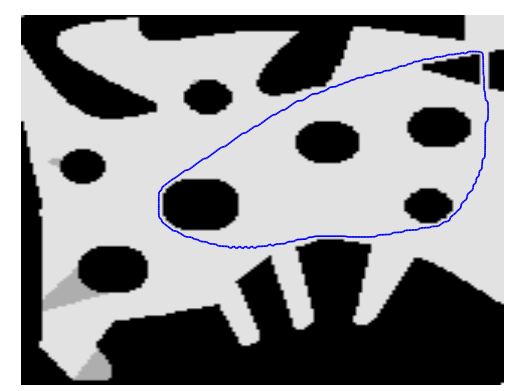

(d) 2000 Iterations

Fig. 3.2: Action of Path Planning Velocity Field on a Curve. 
The initial curve in Figure 3.2 (a) is a simple, closed polygon which was constructed by using the observer positions shown Figure 2.2 (b) as its vertices. Complete visibility coverage of the domain was attained by the initialized curve. In fact, any curve containing these observer positions is guaranteed to attain complete visibility coverage by virtue of its construction. The recent work of Andrews and Sethian [2] on the continuous traveling salesman problem can be used to initialize the curve as the shortest, obstacle avoiding path connecting the observers. However, because of the non-convex nature of this problem the shortest initial path will not necessarily guarantee the shortest final path. As the path planning flow is applied to the curve visibility coverage is lost, as illustrated in Figure 3.2 (b)-(d).

Until now our discussion on the watchman route problem has been somewhat disconnected. We have discussed the visibility requirement and introduced $\vec{v}_{v i s}$, the velocity field which corresponds to the gradient of visibility coverage. We have also discussed the path planning objectives and their corresponding gradient descent velocity field $\vec{v}_{p}$. Finally, we have just mentioned how to initialize a curve which attains complete visibility coverage. Together, these three ingredients suggest the following solution to the watchman route problem:

Initialize a curve that attains complete visibility coverage of the domain and evolve it in the direction which minimizes its length and intersections with obstacles, as long as this evolution does not reduce visibility coverage.

More precisely, the curve will be forced to evolved in the positive direction of the visibility velocity field $\vec{v}_{v i s}$. If the initialized curve attains complete visibility coverage then this is equivalent to evolving the curve in the null direction of $\vec{v}_{v i s}$. However, if the initialized curve does not achieve complete visibility coverage then evolving in the positive direction of $\vec{v}_{v i s}$ will insure that visibility coverage may increase but will never decrease. The resulting projected velocity field that will act on the curve can be expressed as

$$
\vec{v}_{p r o j}=\vec{v}_{p}-H\left(-\vec{v}_{v i s} \cdot \vec{v}_{p}\right) \vec{v}_{p}
$$

Notice that $\vec{v}_{p r o j}=0$ if $\vec{v}_{v i s}$ has a component in the negative direction of $\vec{v}_{p}$. This means that those portions of the curve which are necessary for maintaining visibility coverage on some subset of the domain will remain fixed.

Evolving a curve under (3.10) will ensure that visibility coverage is preserved throughout the evolution while minimizing the curve's length and its intersections with the obstacles. This evolution is depicted in Figure 3.3. The initial curve attains complete visibility coverage and is identical to the initial curve in Figure 3.2 (a). However, unlike the evolution depicted in Figure 3.2 the curve in Figure 3.3 maintains complete visibility coverage throughout its evolution.

Since the action of projecting $\vec{v}_{p}$ in the positive direction of $\vec{v}_{v i s}$ can cause the curve to remain fixed at some locations, it is natural to ask whether this will prevent the minimization of the obstacle avoidance and path length objectives. Intuitively, it is clear that complete visibility coverage cannot be attained by an arbitrarily short curve, therefore we expect that $\vec{v}_{\text {proj }}$ will prevent the curve from contracting at those locations which are necessary for maintaining visibility coverage. We also reason that since the visibility coverage attained by an observer passing through an occluder is zero, visibility coverage cannot be reduced if any part of the curve is moved outside an occluder. Therefore, we expect that the action of projecting $\vec{v}_{p}$ in the positive direction of $\vec{v}_{v i s}$ will not hinder the minimization of the obstacle avoidance objective. 


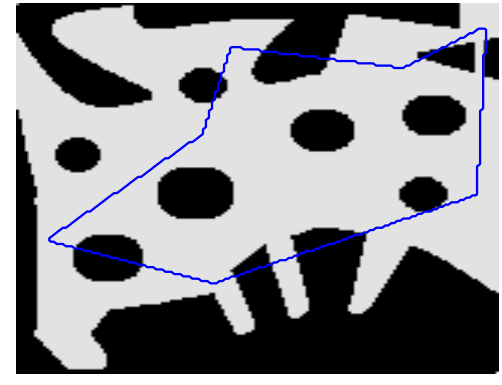

(a) Initial

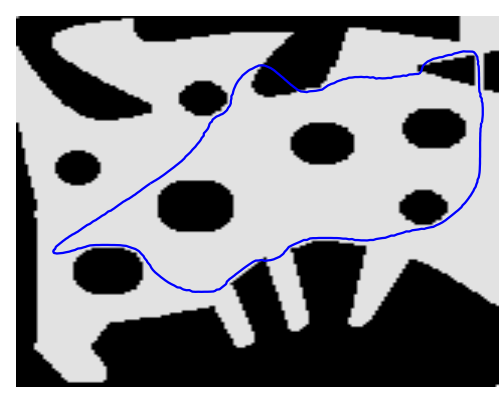

(c) 100 Iterations

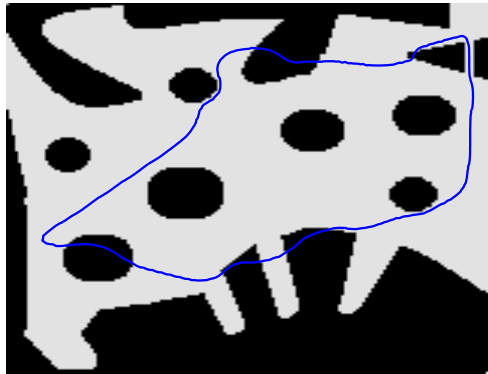

(b) 50 Iterations

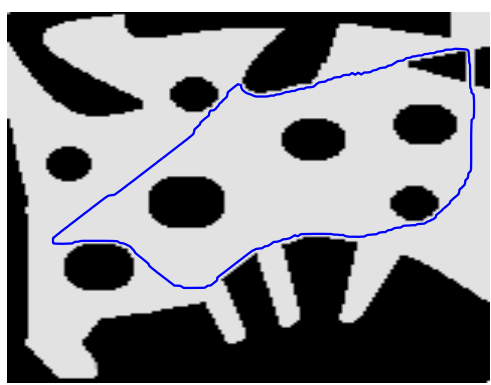

(d) 2000 Iterations

Fig. 3.3: Action of Projected Velocity Field on a Curve.

We will now analyze the behavior of $\vec{v}_{\text {proj }}$ more closely in order to analytically derive these properties.

THEOREM 3.3. The action of projecting $\vec{v}_{p}$ into the positive direction of $\vec{v}_{v i s}$ will not prevent minimizing intersections with occluders.

Proof. We begin by evaluating the term $\vec{v}_{v i s} \cdot \vec{v}_{p}$ in (3.10) which leads to

$$
\begin{aligned}
\vec{v}_{v i s} \cdot \vec{v}_{p} & =\vec{v}_{v i s} \cdot H(-\Psi)([\nabla \Psi \cdot \vec{N}] \vec{N}-\Psi \kappa \vec{N})+\vec{v}_{v i s} \cdot H(\Psi) \kappa \vec{N} \\
& =\vec{v}_{v i s} \cdot H(\Psi) \kappa \vec{N}
\end{aligned}
$$

We have simplified the above expression using the fact that $\vec{v}_{v i s}=0$ inside the occluders (Lemma 2).

Using (3.11) and the orthogonality property $H(-\Psi) H(\Psi)=0$, we can simplify (3.10) as follows:

$$
\begin{aligned}
\vec{v}_{\text {proj }} & =\vec{v}_{p}-H\left(-\vec{v}_{v i s} \cdot H(\Psi) \kappa \vec{N}\right) \vec{v}_{p} \\
& =\vec{v}_{p}-H(\Psi) H\left(-\vec{v}_{v i s} \cdot \kappa \vec{N}\right) \vec{v}_{p} \\
& =H(-\Psi)([\nabla \Psi \cdot \vec{N}] \vec{N}-\Psi \kappa \vec{N})+H(\Psi)\left(\kappa \vec{N}\left[1-H\left(-\vec{v}_{v i s} \cdot \kappa \vec{N}\right)\right]\right) .
\end{aligned}
$$

From the above expression it is clear that $\vec{v}_{\text {proj }}$ consists of two orthogonal components: the first component is the velocity field inside the occluders $(H(-\Psi)$ term) and the second component is the velocity field outside the occluders $(H(\Psi)$ term). The 
velocity field inside the occluders is independent of $\vec{v}_{v i s}$ and can be recognized as the gradient descent on occluder intersection (Equation 3.7). This shows that occluder intersections can always be reduced without interfering with visibility coverage. On the other hand, we see that the second term will be equal to zero (the curve will remain fixed) if $\vec{v}_{v i s}$ has a component in the negative direction of $\kappa \vec{N}$. The second term shows that the curve cannot be made arbitrarily short outside the occluders without losing visibility coverage.

\section{Numerical implementation}

We now describe methods for the numerical computation of equations (3.5), (3.9), and (3.10). The level set evolution equation used to evolve the inspection route and the appropriate finite difference approximation schemes will also be discussed. The proceeding implementation is not the most computationally efficient, however for the purposes of this work we use the simplest implementation to illustrate our solution.

There are three level set functions involved in the implementation, specifically: $\Psi$, the signed-distance embedding of the occluders, $\Phi$, the visibility level set function obtained via the fast sweeping approximation of Equation 1.1, and $P$, the signed distance embedding of the inspection route curve $C$. Assuming that the domain containing the occluders is discretized into an $n \times m$ Cartesian grid, $\Phi$, obtained via fast sweeping, is also $n \times m$ [14]. For simplicity, the inspection route embedding $P$ will be the same size. Note that $\Psi$ and $\Phi$ are static level set functions and only $P$ is evolved. For reasons of numerical implementability similar to those cited in [11] we use the arctangent function and its derivative to approximate all Heaviside and delta functions.

$$
\begin{array}{r}
H(x)=\left\{\begin{array}{lr}
0 & x<-\epsilon, \\
\frac{1}{2}+\frac{2}{\pi} \arctan \left(\frac{x}{\epsilon}\right) & -\epsilon \leq x \leq \epsilon, \\
1 & x>\epsilon,
\end{array}\right. \\
\delta(x)=\left\{\begin{array}{lr}
0 & x<-\epsilon, \\
\frac{1}{1+\left(\frac{x}{\epsilon}\right)^{2}} & -\epsilon \leq x \leq \epsilon, \\
0 & x>\epsilon .
\end{array}\right.
\end{array}
$$

One of the leading reasons for utilizing the level set formulation in this problem is for accurate numerical computation of the exposure line integral $\mathcal{X}(y ; C)$ in Equation (3.5). The line integral is computed by obtaining an explicit (control point) representation of the curve from the level set representation. Converting from the level set representation to the control point representation ensures that the curve remains well represented throughout its evolution. That is, as the curve stretches and contracts, the control points obtained from the implicit representation remain equally spaced. In this way, we have combined the convenience of the level set representation with the accuracy of an explicit representation. In order to reduce the computational complexity of computing the exposure line integral, the visibility level set functions at all observer locations are computed prior to the route evolution and stored in an $(n \times m) \times(n \times m)$ matrix. The visibility level set functions at the vantage points located on the route are interpolated from the precomputed visibility level set functions stored in the matrix. This is the same matrix that was used to reduce the computational complexity of the exhaustive search discussed in Section 2. As described in [4] central differences are used to compute the $\nabla_{x_{0}} \Phi(y ; C(s))$ term. The level set evolution equation is used to implement the curve evolution described by Equation 3.5 (Figure 3.1). 
If the inspection route is embedded in a level set function $P$, then the evolution of the inspection route by the visibility velocity field $\vec{v}_{v i s}$ is given by the level set evolution equation [11]:

$$
P_{t}+\vec{v}_{v i s} \cdot \nabla P=0
$$

Because Equation 3.5 is an advective flow, upwind differences of $\nabla P$ must be computed with respect to $\vec{v}_{v i s}[12,11]$. For example, if $P_{x}$ is the spatial derivative of $P$ in the $x$-direction, $v_{v i s_{x}}$ is the $x$-component of the visibility velocity field, and $\Delta x$ is the grid size, then $P_{x}$ is approximated as

$$
P_{x}(x, t)= \begin{cases}\frac{P(x+\Delta x, t)-P(x, t)}{\Delta x} & v_{v i s_{x}}(x, t)<0, \\ \frac{P(x, t)-P(x-\Delta x, t)}{\Delta x} & v_{v i s_{x}}(x, t)>0 .\end{cases}
$$

An analogous formula is used to compute $P_{y}$.

The level set evolution equation, $P_{t}+\vec{v}_{p} \cdot \nabla P=0$, corresponding to $\vec{v}_{p}$ (Equation 3.9 and Figure 3.2) can be simplified $[11,12]$ to

$$
P_{t}+H(-\Psi) \nabla \Psi \cdot \nabla P+\nabla \cdot \frac{\nabla P}{|\nabla P|}|\nabla P|(H(\Psi)-H(-\Psi) \Psi)=0 .
$$

In the above expression central differences are used to compute $\nabla \Psi$. As prescribed in $[12,11]$, upwind differences of $\nabla P$ must be computed with respect to $H(-\Psi) \nabla \Psi$ and central differences can be used to compute the remaining (curvature) term.

Finally, Equations 4.1 and 4.2 can be combined to implement the level set evolution equation corresponding to 3.10 (Figure 3.3):

$$
P_{t}+\left(\vec{v}_{p}-H\left(\left(-\vec{v}_{v i s} \cdot \nabla P\right)\left(\vec{v}_{p} \cdot \nabla P\right)\right) \vec{v}_{p}\right) \nabla P=0 .
$$

If the level set evolution equations corresponding to $\vec{v}_{p}$ and $\vec{v}_{v i s}$ have already been implemented, then the projection of $\vec{v}_{p}$ in the positive direction of $\vec{v}_{v i s}$ can be computed by reusing the terms in Equations 4.1 and 4.2. However, this approach is not the most computationally efficient because it requires the computation of upwind differences of $P$ with respect $\vec{v}_{v i s}$ and $\vec{v}_{p}$.

\section{Conclusion}

In this work, we have presented solutions to two visibility-based optimization problems. An iterative greedy algorithm was discussed as the solution to the art gallery problem. This solution is less prone to local maxima when compared to gradient based approaches. It was shown how this solution can be used to initialize a curve which attains complete visibility coverage of the domain. A gradient projection strategy was proposed for evolving the curve in a way which minimizes the cost associated with other constraints while preserving visibility coverage. In this way, our solution to the watchman route problem is guaranteed to attain complete visibility coverage of the domain. Our solutions make use of the continuous visibility level-set function developed in [14], and were inspired by the variational approaches introduced in [4]. The novel contribution of our work is the introduction of a framework which generates solutions that attain complete visibility coverage of the domain. In future works more detailed convexity analysis of the functionals involved will be presented in order to identify local minima specific to this problem. We will also make use of global 
optimization search strategies, and propose more efficient strategies for numerically computing the quantities involved.

The algorithms presented in this work can be directly extended into the threedimensional setting. The three-dimensional visibility level set function and the fast sweeping algorithm used to compute it have already been developed in [14]. However, the computational cost of performing exhaustive searches in higher dimensions may be infeasible or must be mitigated by implementing multi-scale techniques. Other generalizations of interest include observers with limited range and field of view.

\section{REFERENCES}

[1] P. K. Agarwal and M. Sharir, Ray shooting amidst convex polygons in 2D, J. Algo., 21(3), 508-519, 1996.

[2] J. Andrews and J. Sethian, Fast marching methods for the continuous traveling salesman problem, Proc. Nat. Aca. Sci., 2007.

[3] V. Caselles, R. Kimmel and G. Sapiro, Geodesic active contours, Int. J. Comput. Vis., 22(1), 61-79, 1997.

[4] L.T. Cheng and Y.H. Tsai, Visibility optimization using variational approaches, Commun. Math. Sci., 3(3), 425-451, 2005.

[5] W.P. Chin and S. Ntafos, Shortest watchman routes in simple polygons, Disc. Comput. Geo., 6(1), 9-31, 1991.

[6] D. Johnson, Approximation algorithms for combinatorial problems, J. Comput. Syst. Sci., 9(3), 256-278, 1974.

[7] Y. Landa and R. Tsai, Visibility of point clouds and exploratory path planning in unkown environments, Commun. Math. Sci., 6(4), 881-913, 2008.

[8] S.M. LaValle, Planning Algorithms, Cambridge University Press, 2006.

[9] S. Makrinos, United States port security in the war on terrorism, Sea Technology, 45(3), 2004.

[10] J. O'Rourke, Art Gallery Theorems and Algorithms, Oxford University Press, Inc., 1987.

[11] S. Osher and R. Fedkiw, Level Set Methods and Dynamic Implicit Surfaces, Springer, 2003.

[12] J. Sethian, Level Set Methods, Cambridge University Press, 1996.

[13] X. Tan, Approximation algorithms for the watchman route and zookeeper's problems, Disc. Appl. Math., 136(2-3), 363-376, 2004.

[14] Y.H.R. Tsai, L.T. Cheng and S. Osher, Visibility and its dynamics in a PDE based implicit framework, J. Comput. Phys., 199(1), 260-290, 2004. 